\title{
Pearls for Treating Retinoblastoma
}

\author{
Sona N Shah ${ }^{1,2}$ and Jesse L Berry ${ }^{1,2}$ \\ 1. Children's Hospital Los Angeles, Los Angeles, CA, US; 2. University of South California (USC) Roski Eye Institute, University of Southern California \\ Keck School of Medicine, Los Angeles, CA, US
}

DOI: https://doi.org/10.17925/USOR.2018.11.2.79

$\mathrm{R}$ etinoblastoma is a primary intraocular cancer that develops in the eyes of children. The vast majority of retinoblastoma tumorigenesis is initiated by a mutation in the RB1 gene on chromosome 13q. This practice pearl will discuss the current treatment modalities for this pediatric tumor, with the primary goal of saving the child's life. With recent treatment advances, more emphasis has been placed on successfully saving the eye, often with useful vision.

\section{Keywords}

Retinoblastoma, pediatric cancer, ocular oncology, RB1, enucleation, chemotherapy, intra-arterial chemotherapy, intravitreal chemotherapy

Disclosure: Sona N Shah and Jesse L Berry have received funding from the Knights Templar Eye Foundation, The Larry and Celia Moh Foundation, The Institute for Families, Inc., and the Children's Hospital Los Angeles, and have an unrestricted departmental grant from Research to Prevent Blindness National Cancer Institute of the National Institutes of Health Award Number K08CA232344.

Review Process: This article is a short educational piece and has not been submitted to external peer reviewers, but was reviewed by the editorial board for accuracy before publication.

Authorship: All named authors meet the International Committee of Medical Journal Editors (ICMJE) criteria for authorship of this manuscript, take responsibility for the integrity of the work as a whole, and have given final approval to the version to be published.

open Access: This article is published under the Creative Commons Attribution Noncommercial License, which permits any noncommercial use, distribution, adaptation, and reproduction provided the original author(s) and source are given appropriate credit. () The Author 2018.

Received: July 25, 2018

Published Online: October 15, 2018

Citation: US Ophthalmic Review. 2018;11(2):79-80

Corresponding Author: Jesse L Berry, USC

Roski Eye Institute, University of Southern

California Keck School of Medicine, CHL 4650

Sunset Blvd, Off Campus, Los Angeles, CA, US.

E: jesse.berry@med.usc.edu

Instagram: @ moda_md

Support: No funding was received in

the publication of this article.
Retinoblastoma is a primary intraocular cancer that develops in the eyes of children. The vast majority of retinoblastoma tumorigenesis is initiated by a mutation in the RB1 gene on chromosome 13q, which was the first tumor-suppressor gene described. Retinoblastoma is a rare disease occurring in approximately one in 18,000 live births annually and can affect one or both eyes. ${ }^{2} \mathrm{~A}$ little over 100 years ago retinoblastoma was usually fatal; however, with the advancements in treatment modalities, the survival rate for these patients is now $>95 \%$ in the developed world. ${ }^{3}$ Treatment must be initiated promptly and the goals of therapy are always: save the life of the child; save the eye(s) of the child, when it is not posing risk to the child's life; and spare as much useful vision as possible. Current treatment options include enucleation, systemic chemotherapy, intra-arterial chemotherapy, and adjunctive local therapies such as cryotherapy, photocoagulation, and brachytherapy. In the past 5 years, intravitreal chemotherapy has emerged as an additional consolidative modality which has mostly replaced the role of eternal beam radiation for retinoblastoma.

\section{Historical treatments}

In the early 20th century enucleation (i.e., surgical removal of the eye) was the only known therapy for retinoblastoma. However, the children were often diagnosed at such advanced stages of the disease that even with enucleation, the rates of metastatic disease were high, and many of these children succumbed to the disease. By the 1940s, radiation (either as external beam radiation or plaque brachytherapy) became the mainstay of therapy and offered the first chances of globe salvage for children with retinoblastoma. However, the long-term sequelae of radiation treatment including bony orbital deformities and, more so, secondary radiation-induced cancers, led clinicians to search for alternative therapies. This ushered in the chemotherapy era in the 1990s. Chemotherapy, in some form, continues to serve as the backbone for modern treatment regimens for retinoblastoma.

It should be noted that while commonalities exist, there are no consensus guidelines for the treatment of retinoblastoma, and regimens differ among centers based on the age of the patient, laterality of the disease, stage/classification of the tumor, and accepted practice patterns.

\section{Systemic chemotherapy}

Generally, systemic chemotherapy regimens consist of carboplatin, vincristine, and etoposide, administered in three to six cycles given monthly. This treatment modality has a $90-100 \%$ cure rate for smaller tumors (groups A-C), but less than a $50 \%$ salvage rate for group D eyes without other consolidative therapies such as previous external beam radiation therapy (EBRT), or intravitreal chemotherapy in the current era. ${ }^{4}$ Risks of intravenous chemotherapy include bone marrow suppression leading to clinically significant cytopenia, secondary leukemia, hearing loss, and neuropathy. This treatment is often used for bilateral retinoblastoma, with a classification of group B or worse in the least affected eye.

\section{Intra-arterial chemotherapy-ophthalmic artery chemosurgery}

During the early 21st century in the United States, based on the work of Yamane et al., ${ }^{5}$ intra-arterial chemotherapy was pioneered for the treatment of retinoblastoma. The procedure 
involves the infusion of chemotherapy (melphalan, topotecan, and carboplatin) into the ophthalmic artery (via a catheter in the femoral artery). The goal is to increase the concentration of chemotherapy in the globe to improve salvage while decreasing the systemic toxicity. Chemotherapy is generally administered over three cycles, and the results are techniquedependent, requiring the expertise of an interventionalist. ${ }^{6}, 7$

This treatment modality has cure rates greater than 90\% reported for primary therapy in group $D$ eyes, and greater than $50 \%$ for recurrent disease following alternate therapies (most often systemic chemotherapy). It is a well-accepted, effective therapy for patients aged over 6 months, patients requiring primary treatment for unilateral group $\mathrm{C}$ or $\mathrm{D}$ retinoblastoma, and patients who need salvage therapy for retinal and vitreous recurrences. ${ }^{8}$ As primary therapy, intra-arterial chemotherapy offers improved globe salvage rates compared to systemic chemotherapy, with lower rates of bone marrow suppression but higher rates of local side effects. Transient local complications include periorbital edema, skin erythema, and nasal lash loss, while rare complications include avascular retinopathy, sectoral choroidal vascular occlusion, and stroke. Whether the risk of metastasis is increased remains a highly debated topic.

\section{Local therapies-cryotherapy, photocoagulation, and brachytherapy}

Whether a child is treated with systemic chemotherapy or intra-arterial chemosurgery, local therapies are used to consolidate the tumors. These include cryotherapy, photocoagulation, and brachytherapy. Cryotherapy is the best modality for small, peripheral tumors, and acts through inducing apoptosis. Photocoagulation is effective for posterior tumors less than $3 \mathrm{~mm}$ in size and can be administered via an argon laser or diode laser. If the tumor is too large for cryotherapy and photocoagulation, brachytherapy may be used; however, radiation retinopathy is a known side effect.9 Group A and some B tumors (peripheral lesions) can be treated with laser or cryotherapy alone with good outcomes.

\section{Intravitreal chemotherapy injection}

Intravitreal chemotherapy was introduced initially in the 1990s to treat vitreous seeding, a common and aggressive cause of tumor relapse, but was quickly abandoned due to safety concerns regarding extraocular spread. ${ }^{10}$ Then in 2012 Francis Munier described a case series of patients treated with a safety-enhanced procedure for intravitreal injections of chemotherapy. ${ }^{11}$ This new treatment approach involves the administration of chemotherapy (most often melphalan) intravitreally as salvage treatment for eyes that display refractive or recurrent vitreous seeding. As an initial safety mechanism, a paracentesis is performed withdrawing $0.1 \mathrm{cc}$ of aqueous humor to lower the intraocular pressure and prevent reflux of active seeds to the injection site. The chemotherapy injection can then be performed in a quadrant of the eye that is confirmed free of tumor by ultrasound biomicroscopy. The needle is placed into the vitreous cavity via the pars plana and visualized behind the lens during injection. Cryotherapy is then applied at the injection site as the needle is withdrawn. The eye is shaken to distribute the chemotherapy, and the surface is bathed in sterile water which is cytotoxic. The known toxicities of this treatment modality are retinal pigment epithelium mottling, hypotony, anterior segment inflammation, and cataract formation; however, rarely severe complications such as maculopathy, optic neuropathy, and phthisis can occur. ${ }^{12}$ The efficacy for treating vitreous seeds with intravitreal injections has been reported to be up to $100 \%$ at many centers ${ }^{11}$ and has enabled many children to be successfully treated without EBRT.

\section{Enucleation}

With improved efficacy of retinoblastoma treatment modalities, the focus is often on globe salvage for these patients. However, enucleation remains an important tool for the ocular oncologist. Enucleation is a 1-hour surgery which can be used as primary therapy for advanced unilateral eyes with poor vision potential such as those with diffuse disease, significant vitreous, or subretinal seeding (group D), or any eyes with one or more of the following poor prognosis features (group E): tumor touching the lens, tumor anterior to the anterior vitreous face involving ciliary body or anterior segment, diffuse infiltrating retinoblastoma, neovascular glaucoma, opaque media from hemorrhage, tumor necrosis, or phthisis bulbi. The intent, of course, is a surgical cure which is successful for $>96 \%$ of unilateral cases. ${ }^{13}$ Enucleation is also used for eyes that have persistent or recurrent disease poorly responsive to therapy. This is called secondary enucleation.

\section{Conclusion}

The approach to retinoblastoma treatment has transformed dramatically over the past 100 years. Each modality carries its own risks and benefits, but our job as pediatric ocular oncologists is to understand when to best use which treatment based on the patients' clinical features, which include the classification, size, extent, and location of the tumor. While enucleation initially served as our primary form of treatment, the use of chemotherapy and local consolidation techniques now allows us not only to choose from, but also integrate, various methods to effectively target this complex disease. We now have the ability to save an unprecedented number of eyes, many with useful vision. The next step in retinoblastoma management will surely be personalized medicine for retinoblastoma based on tumor biomarkers. This has historically been limited by the inability to biopsy this tumor for fear of tumor spread. However, recent advances in using the aqueous humor as a liquid biopsy for retinoblastoma show promise in overcoming this barrier. ${ }^{14}$ Regardless of the treatment approach used for retinoblastoma, the goals for these patients remain the same: saving the life of the child first, saving the eye when safe to do so, and sparing as much useful vision as possible during the course of treatment. $]$
1. Friend SH, Bernards R, Rogelj S, et al. A human DNA segment with properties of the gene that predisposes to retinoblastoma and osteosarcoma. Nature. 1986,323:643-6.

2. Peylan-Ramu N, Bin-Nun A, Skleir-Levy M, et al. Orbital growth retardation in retinoblastoma survivors: work in progress. Med Pediatr Oncol. 2001;37:465-70.

3. Broaddus E, Topham A, Singh AD. Survival with retinoblastoma in the USA: 1975-2004. Br J Ophthalmol. 2009;93:24-7.

4. Zhu D, Berry JL, Ediriwickrema L, et al. Long-term outcomes of Group $B$ eyes in patients with retinoblastoma treated with short-course chemoreduction: experience from Children's Hospital Los Angeles/University of Southern California. Ocul Oncol Pathol. 2015;2:105-11.

5. Yamane T, Kaneko A, Mohri M. The technique of ophthalmic arterial infusion therapy for patients with intraocular retinoblastoma. Int J Clin Oncol. 2004;9:69-73.

6. Shields CL, Alset AE, Say EA, et al. Retinoblastoma control with primary intra-arterial chemotherapy: outcomes before and during the intravitreal chemotherapy era. J Pediatr Ophthalmol Strabismus. 2016;53:275-84.

7. Shields $\mathrm{CL}$, Manjandavida FP, Lally $\mathrm{SE}$, et al. Intra-arterial chemotherapy for retinoblastoma in 70 eyes: outcomes based on the international classification of retinoblastoma. Ophthalmology 2014;121:1453-60

8. Shields CL, Lally SE, Leahey AM, et al. Targeted retinoblastoma management: when to use intravenous, intra-arterial, periocular, and intravitreal chemotherapy. Curr Opin Ophthalmol. 2014;25:374-85.

9. Chawla B, Jain A, Azad R. Conservative treatment modalities in retinoblastoma. Indian J Ophthalmol. 2013;61:479-85.

10. Kaneko A, Suzuki S. Eye-preservation treatment of retinoblastoma with vitreous seeding. Jpn J Clin Oncol. 2003;33:601-7.

11. Munier FL, Gaillard MC, Balmer A, et al. Intravitreal chemotherapy for vitreous disease in retinoblastoma revisited: from prohibition to conditional indications. Br J Ophthalmol. 2012;96:1078-83.

12. Aziz HA, Kim JW, Munier FL,Berry JL. Acute hemorrhagic retinopathy following intravitreal melphalan injection for retinoblastoma: a report of two cases and technical retinoblastoma: a report of two cases and technical
modifications to enhance the prevention of retinal toxicity. Ocul Oncol Pathol. 2017;3:34-40.

13. Kim JW, Kathpalia V, Dunkel IJ, et al. Orbital recurrence of retinoblastoma following enucleation. Br J Ophthalmol. 2009;93:463-7.

14. Berry JL, Xu L, Murphree AL, et al. Potential of aqueous humor as a surrogate tumor biopsy for retinoblastoma. JAMA Ophthalmol. 2017;135:1221-30. 